\title{
Redes, tweets y engagement: análisis de las bibliotecas universitarias españolas en Twitter
}

\section{Networks, tweets and engagement: Analysis of Spanish university libraries on Twitter}

\author{
Rafael Carrasco-Polaino; Ernesto Villar-Cirujano; Miguel-Ángel Martín-Cárdaba
}

Cómo citar este artículo:

Carrasco-Polaino, Rafael; Villar-Cirujano, Ernesto; Martín-Cárdaba, Miguel-Ángel (2019). "Redes, tweets y engagement: análisis de las bibliotecas universitarias españolas en Twitter". El profesional de la información, v. 28, n. 4, e280415.

https://doi.org/10.3145/epi.2019.jul.15

Artículo recibido el 09-03-2019

Aceptación definitiva: 11-06-2019

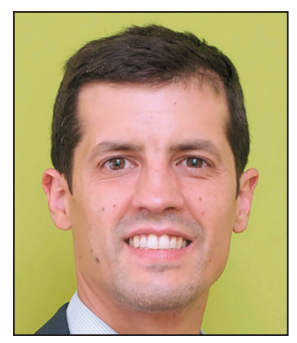

Rafael Carrasco-Polaino $\bowtie$ https://orcid.org/0000-0002-0687-6842

Universidad Complutense de Madrid Departamento de Periodismo y Nuevos Medios

Av. Complutense, 3.

28040 Madrid, España

racarras@ucm.es

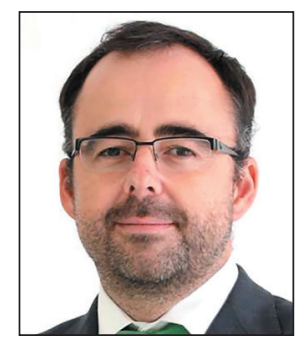

Ernesto Villar-Cirujano https://orcid.org/0000-0001-6263-5093

Centro Universitario Villanueva Costa Brava, 2. 28034 Madrid, España evillar@villanueva.edu

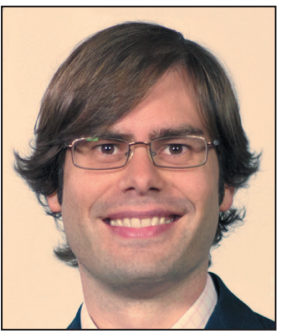

Miguel-Ángel Martín-Cárdaba https://orcid.org/0000-0003-3897-2537

Centro Universitario Villanueva

Costa Brava, 2. 28034 Madrid, España

mmartincar@villanueva.edu

\section{Resumen}

Las bibliotecas de las universidades españolas han aumentado significativamente su presencia y su actividad en las redes sociales, especialmente en Twitter. En el presente artículo se analiza el uso que las bibliotecas pertenecientes a la Red de Bibliotecas Universitarias Españolas (Rebiun) realizan de Twitter. En concreto, a través del análisis de redes sociales, de un análisis de contenido y de pruebas estadísticas no paramétricas, esta investigación analiza la red generada en Twitter por las 59 bibliotecas universitarias españolas que tienen perfil en esta plataforma, así como la finalidad, el contenido, el formato y el engagement (compromiso, fidelidad) de sus tweets. Los resultados obtenidos indican que las bibliotecas universitarias conforman en Twitter una red de un solo componente con cierto grado de cohesión, pero de varias comunidades, en la que los tweets de información de servicio y los que tienen imagen son los más numerosos. Los tweets con imágenes generan mayor engagement independientemente de su contenido.

\section{Palabras clave}

Twitter; Bibliotecas universitarias; Engagement; Redes de nodos; Implicación; Compromiso; Participación; Redes sociales; Medios sociales; Enlaces; Contactos; Participación; Colaboración; Rebiun.

\section{Abstract}

The libraries of Spanish universities have significantly increased their presence and activity on social networks, especially on Twitter. This article analyzes the use that Spanish university libraries (member of the Red de Bibliotecas Universitarias Españolas, Rebiun) make of Twitter. Specifically, through the analysis of social networks, content analysis and non-parametric statistical tests, this research analyzes the network generated on Twitter by the 59 Spanish university libraries that 
have profiles on this platform as well as the purpose, content, the format and engagement of their tweets. The results indicate that the university libraries form a single-component network on Twitter with a certain degree of cohesion, but with several communities, in which the tweets about service information and the tweets with pictures are the most numerous. Tweets with pictures generate greater engagement independently of their content.

\section{Keywords}

Twitter; University libraries; Engagement; Node networks; Social media; Participation; Collaboration; Links; Contacts; Relations.

\section{Introducción}

Las bibliotecas, que tradicionalmente han actuado como guardianes y difusores del conocimiento (Curran et al., 2006), han experimentado cómo el desarrollo de nuevas tecnologías ha facilitado y potenciado su capacidad de recolectar, almacenar y compartir dicho conocimiento. Hoy en día, desde que gracias a internet y a la digitalización las bibliotecas ya no sufren las limitaciones propias del espacio y las estanterías han sido reemplazadas por catálogos en una pantalla, la distancia entre la biblioteca y el usuario se ha reducido de modo radical (Wallis, 2007). La misión fundamental de las bibliotecas, por tanto, se ha mantenido, pero los procesos, las herramientas y las técnicas han experimentado una enorme evolución (Holmberg et al., 2009).

Con internet y la digitalización, las bibliotecas ya no sufren las limitaciones propias del espacio, y las estanterías han sido reemplazadas por catálogos en una pantalla

Uno de los elementos más relevantes de esta transformación tecnológica ha sido la llegada de la web 2.0. Este término, popularizado por O’Reilly (2005), se utiliza para hacer referencia al conjunto de todas las nuevas tecnologías (redes sociales, blogs, plataformas de fotografías y vídeos, etc.) que permiten la personalización y, sobre todo, la interacción entre webs, y de las que las redes sociales son la máxima expresión.

La existencia de estas nuevas tecnologías y la creciente popularidad de las redes sociales han hecho reflexionar a las bibliotecas sobre las múltiples posibilidades que ofrecen y el modo en el que podrían introducir dichos medios comunicativos tanto para potenciar su función original como para promocionarse aún más en sus comunidades, siendo precisamente las bibliotecas unas de las primeras instituciones de cualquier índole en incorporarse al entorno de la web 2.0 (Marcos, 2009; Margaix-Arnal, 2007; Miller, 2005; Stephens, 2007). Dicha aplicación de las nuevas tecnologías web interactivas, colaborativas y multimedia a las actividades y servicios bibliotecarios ha recibido la denominación de "biblioteca 2.0" (Casey; Savastinuk, 2007; Connor, 2007; Dickson; Holley, 2010; Maness, 2006; Marquina, 2018).

A pesar de que la adopción de las redes sociales por parte de las bibliotecas ha sido un proceso lento y pausado (Charnigo; Barnett-Ellis, 2007; Booker; Bandyopadhyay, 2013), en la actualidad la mayoría de las universidades añade enlaces a sus redes sociales en su página web principal. La finalidad primordial es no sólo permitir el diálogo fluido con los bibliotecarios respecto a temas relacionados con el uso de la biblioteca, sino también construir relaciones con los usuarios, referenciar servicios o diseminar noticias, así como aumentar la difusión de los programas y actividades de la biblioteca, convirtiendo a estas instituciones en centros de conexión (Ayiah; Kumah, 2011; Jain, 2014; Young; Rossmann, 2015).

Hoy en día, las bibliotecas tienen a su disposición una gran variedad de redes sociales (Facebook, Twitter, Instagram, LinkedIn, YouTube, Flickr, Pinterest, etc.), así como servicios de mensajería muy populares (WhatsApp) o blogs. Pero de todas las redes sociales las dos que utilizan principalmente las bibliotecas a nivel mundial son Facebook y Twitter (Harrison et al., 2017; Sheikh; Kazim-Ali; Naseer, 2016). Este dato encaja, a su vez, con la preferencia que muestran los estudiantes universitarios por recibir información de las bibliotecas precisamente a través de esas dos redes sociales (Howard et al., 2018).

De las dos, Facebook -red social nacida en 2004 dentro de la Harvard University para que los estudiantes intercambiaran información, bajo la premisa de "crear comunidad", y que traspasó las fronteras universitarias hasta el punto de alcanzar un tráfico de 2.232 millones de usuarios a 31 de diciembre de 2018 (Facebook, 2019)- ha sido quizá la red social más empleada hasta la fecha por parte de las bibliotecas universitarias y, por este motivo, en la última década se puede encontrar un creciente número de investigaciones centradas en estudiar la eficacia de posibles usos y utilidades alternativas o complementarias que las bibliotecas podrían hacer de Facebook (Bodnar; Doshi, 2011; Connell, 2009; Glazer, 2012; Laudano et al., 2016; Margaix-Arnal, 2008; Sachs; Eckel; Langan, 2011; Petit, 2011; Phillips, 2015; Wan, 2011).

Por otro lado, respecto a Twitter -red social con más de 230 millones de usuarios activos al mes que publican más de 500 millones de tweets al día (Twitter, 2019) - los últimos datos a nivel mundial sugieren que las bibliotecas universitarias están incrementando notablemente su participación en ella, así como el volumen de tweets y el número de seguidores (Al-Daihani; Abrahams, 2016, Vaughan; Gao, 2016). A causa de esto, el campo de la biblioteconomía ha mostrado también un interés cada vez mayor en estudiar y analizar:

- el modo en el que las bibliotecas universitarias usan Twitter (Del-Bosque; Leif; Skarl, 2012; Emery; Schifeling, 2015; Vassilakaki; Garoufallou, 2015; Shulman et al., 2015); 
- la forma en la que interactúan entre ellas, con sus seguidores y con otros usuarios (Yep; Shulman, 2014; Sewell, 2013; Yep et al., 2017);

- el contenido de sus tweets (Aharony, 2010) o su efectividad (Stvilia; Gibradze, 2014).

Por otra parte, si analizamos el uso que las bibliotecas universitarias de nuestro país hacen de estas dos redes sociales los datos muestran que, hasta hace poco, Facebook ha sido la red más usada y un número elevado de bibliotecas viene participando en esta red desde hace varios años (González-Fernández-Villavicencio, 2007; Grande-González; De-la-Fuente-Redondo, 2012; Herrera-Morillas; Castillo-Díaz, 2011; Castillo-Díaz; Herrera-Morillas, 2014). Sin embargo, igual que ha ocurrido en el panorama internacional, el uso que las bibliotecas universitarias españolas hacen de Twitter ha crecido tanto en los últimos años que se ha equiparado completamente con Facebook. En concreto, según Martín-Marichal (2017), el 73,7\% de las bibliotecas universitarias españolas tiene presencia propia en Facebook y el 77,6\% en Twitter.

Lo que resulta interesante es que mientras que sí pueden encontrarse diversas investigaciones que estudian el uso que las bibliotecas de nuestro país hacen de Facebook (p. ej. Arroyo-Vázquez, 2018), es sorprendente que, hasta donde nosotros sabemos, prácticamente no existe ninguna investigación previa que analice el uso que nuestras bibliotecas universitarias hacen de Twitter, una de las redes sociales más relevantes a nivel mundial.

Por eso, en este trabajo nos proponemos llenar este vacío intentando responder a las siguientes preguntas de investigación, que podríamos articular en torno a cuatro campos:

- En cuanto a la red de bibliotecas españolas en sí misma, ¿qué características tiene? ¿Cuántos componentes la conforman? ¿Qué nivel de cohesión muestran entre sí?

- Respecto a los actores, ¿quiénes son los más influyentes? ¿Son las bibliotecas las que inician las conversaciones o se centran en retweetear contenidos ajenos? ¿Fomentan el diálogo y mantienen activa la red?

- En lo referente a la interacción, ¿qué difusión alcanzan los mensajes? ¿Cómo interactúan, se relacionan y se comunican entre sí los actores?

- Por último, en cuanto a los formatos, los contenidos y la repercusión de los tweets (y sobre todo la relación que tienen estas tres variables entre sí), ¿cuáles son los formatos (texto, imágenes, vídeos) más empleados por las bibliotecas universitarias? ¿Con qué finalidad se utilizan en la plataforma? ¿Las bibliotecas universitarias españolas utilizan una red como Twitter para difundir información útil, para promocionar las actividades de la universidad, para hacer comunidad universitaria o simplemente para compartir conocimiento? ¿Qué características tienen los tweets más difundidos? ¿Cuáles son los tweets que más engagement registran?

\section{Metodología}

Con el objetivo de determinar cómo se conforma la red de bibliotecas universitarias españolas en Twitter, el uso que hacen de la plataforma y la interacción de sus diferentes mensajes en función de su contenido y su forma se llevó a cabo un estudio mediante dos metodologías: análisis de redes y análisis de contenido.

\subsection{Análisis de redes}

Mediante el programa NodeXL Pro (Smith et al., 2010) se recogió toda la actividad (tweets, retweets, respuestas y menciones) generada por las cuentas de Twitter de las 59 bibliotecas universitarias pertenecientes a la Red de Bibliotecas Universitarias Españolas (Rebiun) (CRUE, 2017) y presentes en esta red de microblogging, además de toda la actividad generada por la propia Rebiun durante el curso académico 2017-2018, es decir, entre el 1 de septiembre de 2017 y el 31 de agosto de 2018. Con este mismo programa y aplicando el análisis de redes sociales (ARS) (Lozares, 1996) como metodología, se analizaron las características generales de la red de nodos resultado de todas las menciones en Twitter, así como las características concretas de los nodos (bibliotecas) y aristas (menciones).

En cualquier red algunos usuarios son más importantes que otros. Un aspecto relevante del análisis de las redes sociales es la identificación de la importancia y la influencia de los sujetos en las diferentes redes. Existen muchas definiciones sobre la importancia, cada una de ellas con su propio método de medición, que permite la cuantificación de la relevancia de cada sujeto. En esta investigación se utilizan cuatro métricas, conocidas como medidas de centralidad (Yep et al., 2017):

\section{In-degree}

Uno de los métodos más obvios para determinar la influencia de las cuentas es medir la cantidad de atención que recibe. Esto puede lograrse calculando el número de aristas que le apuntan, en este caso el número de cuentas de usuario que mencionan o responden a la cuenta en cuestión. Es una manera sencilla de medir la popularidad.

\section{Out-degree}

La influencia no se limita a la popularidad. Las cuentas que son proactivas también pueden ser muy influyentes. Quizá una determinada cuenta habla de muchas otras sobre un tema en concreto. Esta cuenta está involucrando a otros, produciendo contenido y manteniendo el tema activo. La influencia de este usuario se puede cuantificar calculando el número de aristas de las que es origen. Este número se conoce como out-degree. Es la cantidad de otras cuentas en la red que una cuenta responde o menciona. 


\section{Eigenvector centrality}

Las métricas de grado valoran cada conexión por igual. Sin embargo, resulta sencillo de entender que conectarse con alguien que ya es importante en la red otorgará más influencia que alguien que no es importante.

Esta medida tiene en cuenta este aspecto adicional. Esta métrica permite que las cuentas se consideren influyentes si muchas otras se conectan a ella y/o sólo algunas cuentas influyentes se conectan a ella.

\section{Betweenness centrality}

Uno no tiene que ser popular, proactivo, ni tener amigos influyentes para ser un miembro importante en una red. Una cuenta que une dos comunidades aisladas puede considerarse influyente ya que la información que viaja entre los dos grupos debe fluir a través de ella. Puede elegir pasar esa información o restringirla. Este es el ejemplo de una cuenta con un alto nivel de intermediación. Dicha cuenta se encuentra en muchos caminos cortos que conectan otras cuentas en una red. En Twitter, la información generalmente viaja en cadenas cortas de menos de seis cuentas (Yang; Counts, 2010). Por lo tanto, aquellos que se encuentran en muchas de estas cadenas cortas tienen la capacidad de controlar el flujo de información en la red.

Los niveles de in-degree y de out-degree tienen significados objetivos en el análisis de redes; son el número total de conexiones entrantes y salientes que posee una cuenta. Por otra parte, los índices eigenvector centrality y betweenness centrality no tienen tales significados. Los valores de estas métricas son relativos y sólo se pueden comparar entre cuentas dentro de la misma red.

\subsection{Análisis de contenido}

Una vez analizada la configuración de la red, se procedió a analizar el contenido y forma de los tweets publicados por las bibliotecas universitarias. Para ello se escogió una muestra aleatoria mediante una aplicación web (Al azar, 2019), de 379 tweets, suficiente para un margen de error del 5\% y una confianza del 95\% en una población de 24.508 tweets originales (sin incluir respuestas, menciones o retweets), según la ecuación del cálculo de muestras para poblaciones finitas (Morales-Vallejo, 2012).

Sobre los tweets de esta muestra se hizo un análisis de contenido (Piñuel-Raigada, 2002) para determinar el tipo de mensaje del tweet según una ficha creada con anterioridad.

La ficha que se elaboró para realizar el análisis de contenido se estructuró en dos apartados. En el primero se identificó el formato del tweet original según las categorías:

- sólo texto (incluidos emoticonos);

- inclusión de imagen (tanto fotografía como GIFs animados);

- inclusión de vídeo.

En un segundo apartado se establecieron categorías en función del contenido del mensaje publicado:

- adquisición y difusión de fondos bibliográficos: la primera pretende dar a conocer a los usuarios la incorporación de un nuevo título a los fondos de la biblioteca; la segunda, animar a la consulta de títulos a raíz de una noticia de actualidad ( $p$. ej. la concesión de un premio a un autor o un acontecimiento relevante) o de una efeméride sobre un tema concreto;

- difusión de tesis y artículos científicos y periodísticos, y entre estos últimos aquellos de temática académica, científica o cultural;

- altavoz de actividades culturales y académicas de la universidad que exceden del ámbito de la biblioteca;

- información de servicio sobre la biblioteca y recursos bibliográficos, así como consejos para la consulta o elaboración de artículos científicos para así mejorar la formación de los estudiantes en este campo;

- difusión de actualidad no universitaria de temática general excluyendo los temas del segundo apartado.

- otros.

Con los datos recogidos se llevó a cabo una prueba de Chi-cuadrado (Rodríguez, 2004) para determinar si se encontraban diferencias significativas entre el contenido del tweet y el formato del mismo.

Posteriormente, con la finalidad de analizar el grado en el que los diferentes tweets son aceptados y difundidos por sus receptores, se realizó un análisis de la varianza Anova (Spiegel; Stephens, 2009) para identificar la posible existencia de diferencias significativas en el engagement de los tweets en relación con su formato y su contenido. El engagement se determinó como la suma de los favoritos y de los retweets de cada tweet, dividido entre el número de seguidores del usuario autor del mismo (Herrera-Torres et al., 2017). Otras investigaciones han utilizado como medida de análisis para objetos de estudio similares los retweets o los favoritos obtenidos por cada tweet (Stvilia; Gibradze, 2014). Sin embargo, se considera que utilizar la medida del engagement puede ofrecer resultados más válidos, ya que pondera y homogeniza la cuantificación de retweets y favoritos, al dividirlos entre el número de seguidores del autor del tweet en el momento de su publicación. 


\section{Resultados}

\subsection{Análisis de la red de nodos}

A Rebiun pertenecen 75 bibliotecas. Con la finalidad de estudiar la composición y forma de la actividad de esta red en Twitter se realizó, mediante NodeXL, un análisis de los tweets publicados por las 59 bibliotecas universitarias con presencia en Twitter pertenecientes a Rebiun, además de los tweets publicados por la propia Rebiun, entre el 1 de septiembre de 2017 y el 31 de agosto de 2018. La red que generó la escucha está formada por 11.660 usuarios que produjeron un total de 87.792 interacciones, siendo 11.513 de ellas únicas y 76.257 duplicadas (usuarios que han interactuado entre sí más de una vez). En concreto, se analizaron 24.508 tweets originales, 42.327 menciones, 18.672 retweets y 2.285 respuestas.

La red de nodos resultante presenta un solo componente (figura 1). Es decir, todos los nodos están conectados a través de otros a todos los demás, con una distancia geodésica máxima (Bouttier; Di-Francesco; Guitter, 2003) de 5 (número de nodos por los que hay que pasar para conectar los más alejados) y una distancia geodésica media de 3,41. La red presenta una densidad del 0,013\% y una modularidad (Newman, 2006) de 0,140092 (solidez de las conexiones que hay entre los vértices de una misma comunidad). Estos datos demuestran la existencia de una única red con un solo componente y no una red en donde existen diferentes grupos independientes y no conectados entre

La red de nodos que conforman las bibliotecas universitarias en Twitter presenta un solo componente. Todos los nodos están conectados a través de otros a todos los demás sí. Por otro lado, una modularidad como la encontrada muestra una cohesión entre los usuarios relativamente fuerte, más allá de la que se encontraría por azar (recordemos que la modularidad se determina entre $-0,5$ y 1 ).

A la hora de analizar los nodos más relevantes, según diferentes índices representativos en el análisis de redes sociales, se identificaron usuarios con una posición importante (tabla 1).

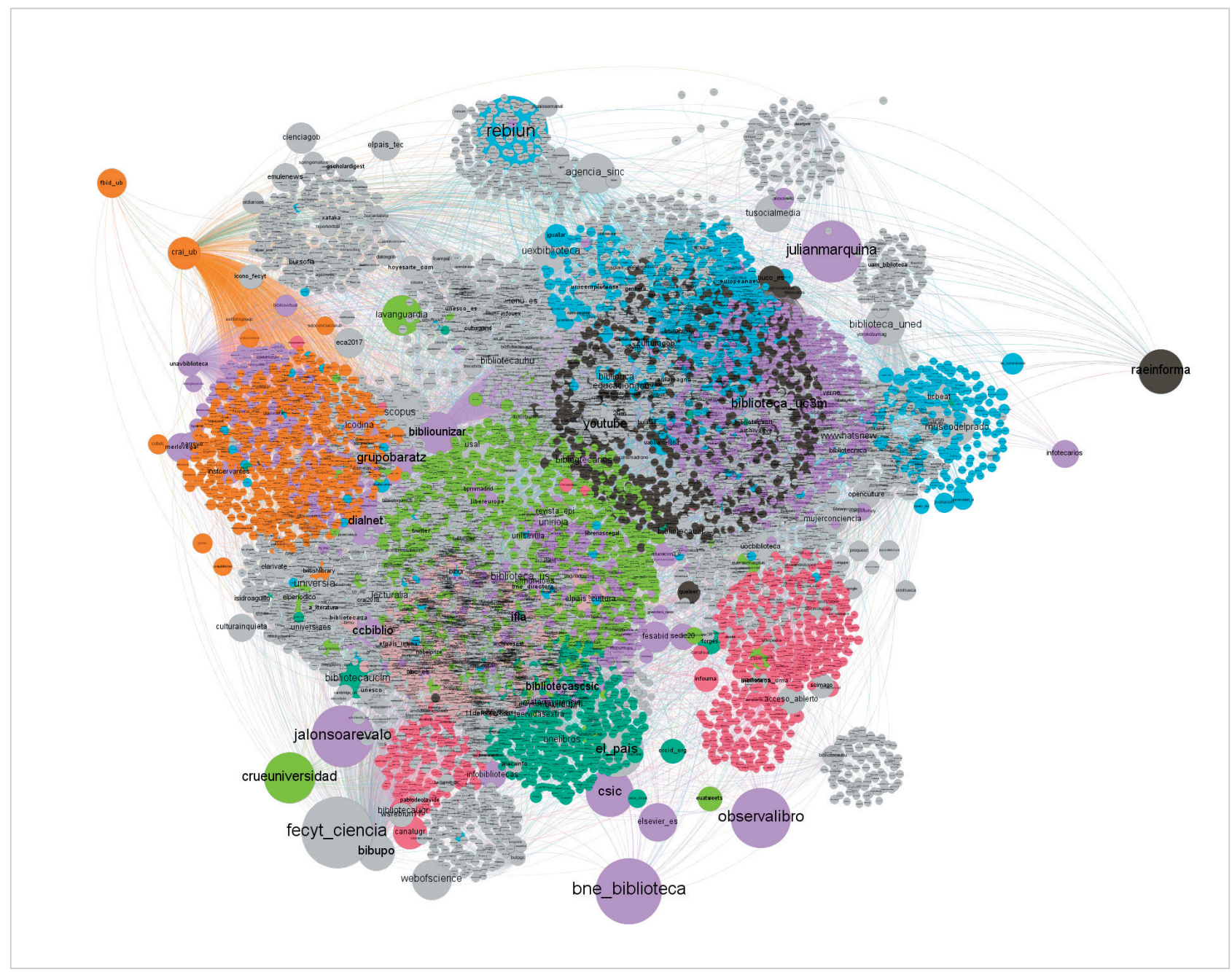

Figura 1. Red de menciones 
Tabla 1. Principales medidas de centralidad

\begin{tabular}{|c|c|c|c|c|c|c|c|c|}
\hline $\begin{array}{l}\text { N. de } \\
\text { orden }\end{array}$ & Usuario & $\begin{array}{l}\text { In-de- } \\
\text { gree }\end{array}$ & Usuario & $\begin{array}{l}\text { Out- } \\
\text { degree }\end{array}$ & Usuario & $\begin{array}{l}\text { Betweenness } \\
\text { centrality }\end{array}$ & Usuario & $\begin{array}{l}\text { Eigenvector } \\
\text { centrality }\end{array}$ \\
\hline 1 & Rebiun & 44 & $\begin{array}{l}\text { Universitat Ramon } \\
\text { Llull (URL) }\end{array}$ & 1.362 & $\begin{array}{l}\text { Universitat Ramon } \\
\text { Llull (URL) }\end{array}$ & 24907780,74 & $\begin{array}{l}\text { Biblioteca CRAI } \\
\text { UPO }\end{array}$ & 0,004604 \\
\hline 2 & Fecyt_Ciencia & 42 & Biblioteca UCO & 1.058 & Biblioteca UCO & 19513543,18 & CRAI UniBarcelona & 0,004447 \\
\hline 3 & $\begin{array}{l}\text { Biblioteca Nacional } \\
\text { de España }\end{array}$ & 39 & CRAI UniBarcelona & 930 & Biblioteca Unizar & 16776637,32 & Biblioteca UCO & 0,004381 \\
\hline 4 & Julio Alonso Arévalo & 36 & Biblioteca Unizar & 902 & CRAI UniBarcelona & 16617566,7 & CSIC - Bib y Arch & 0,004209 \\
\hline 5 & Julián Marquina & 36 & Biblioteca UCLM & 742 & Biblioteca UCLM & 12639403,7 & Biblioteca Unizar & 0,004173 \\
\hline 6 & Observatorio Libro & 35 & Biblioteca CRAI UPF & 681 & Biblio_UM & 11261026,51 & Biblioteca UCLM & 0,004138 \\
\hline 7 & Elpaís & 31 & Biblio_UM & 679 & Biblioteca CRAI UPF & 10830180,27 & Biblioteca UC & 0,003562 \\
\hline 8 & Baratz & 29 & Biblioteca CRAI UPO & 646 & $\begin{array}{l}\text { Biblioteca CRAI } \\
\text { UPO }\end{array}$ & 9375652,048 & Biblio_UM & 0,003248 \\
\hline 9 & CRUE Universidades & 29 & CSIC-Bib y Arch & 549 & CSIC-Bib y Arch & 7807937,044 & $\begin{array}{l}\text { Biblioteca Univer- } \\
\text { sidad de Navarra }\end{array}$ & 0,003218 \\
\hline 10 & CSIC & 27 & UOC biblioteca & 517 & UOC biblioteca & 7689724,23 & $\begin{array}{l}\text { Biblioteca Uni- } \\
\text { versidad Loyola } \\
\text { Andalucía }\end{array}$ & 0,003118 \\
\hline
\end{tabular}

Es significativo que ninguna de las bibliotecas aparezca entre los usuarios más mencionados (in-degree) de la red, lo que denota que cuando las bibliotecas universitarias difunden sus mensajes, ya sea información procedente de otros organismos o instituciones, de la universidad a la que pertenecen o incluso de la propia biblioteca, no generan una interacción por parte del usuario, que generalmente se limita a recibir esa información, pero sin reenviarla.

Por otro lado, cuando se analiza quiénes son los usuarios que más mencionaron a otros usuarios en sus publicaciones (out-degree), los resultados mostraron que esta vez sí son las bibliotecas las que aparecen en las primeras posiciones.

Niveles altos de out-degree denotan el interés de los usuarios por generar conversación e interacción en la red. En este aspecto es relevante la labor que realizan las bibliotecas universitarias españolas, que intentan mantener una reciprocidad en el tráfico de los mensajes.

También las bibliotecas muestran los mayores niveles de betweenness centrality, destacando de forma significativa la de la Universitat Ramon Llull.

Las cuentas más relevantes por sus niveles de eigenvector centrality también se concentran en usuarios de bibliotecas universitarias, destacando por encima de todas la de la biblioteca de la Universidad Pablo de Olavide, de Sevilla, por ser el nodo con mayor número de conexiones relevantes dentro de la propia red.

Al considerar Twitter como una red social en donde los usuarios tienen la posibilidad de interactuar entre sí estableciendo diferentes tipos de relaciones, resulta interesante comprobar cómo las interacciones mutuas entre el total de interacciones (índice de reciprocidad) son muy escasas, tan sólo del 0,88\%.

La tabla 2 muestra el análisis de los grupos de la red.

A través de un algoritmo de detección de comunidades (clusters) dentro de redes de nodos (Clauset; Newman; Moore, 2004), se identificaron 21 grupos en torno a los que se situaban todos los usuarios de la red. Los únicos que siguen un criterio geográfico son el primer grupo, formado por 9 bibliotecas catalanas (más la fronteriza Jaume I de Castellón), y el quinto, con tres centros madrileños. En el resto hay grupos con cierta tendencia a esta vinculación geográfica que no es del todo homogénea (el G6, con universidades de Murcia, Alicante y Oviedo; o el G10, con dos centros canarios al que se une uno valenciano). Estos criterios de proximidad se deben posiblemente a su pertenencia

Resulta relevante la labor que realizan las bibliotecas universitarias españolas, que intentan mantener una reciprocidad en el tráfico de los mensajes

a los consorcios de bibliotecas, una fórmula que permite a centros de distintas zonas geográficas unificar acciones como los préstamos interbibliotecarios, la catalogación o la adquisición coordinada de colecciones (Pérez-Arranz, 2008). Además, existen grupos de usuarios en donde sólo hay una biblioteca, como es el caso de la Europea de Madrid, Complutense, Carlos III, UNED, Granada, Málaga, Cádiz, Ramon Llull, Illes Balears, Córdoba, Zaragoza o Castilla-La Mancha, lo que contribuye a aminorar el peso del criterio geográfico.

\subsection{Análisis de contenido de los tweets originales}

Tras el análisis de la conformación de la red y sus nodos más relevantes, se analizó tanto el contenido (dentro de las categorías mencionadas anteriormente en las que están incluidos los principales tipos de mensaje que las bibliotecas 
Tabla 2. Agrupaciones de bibliotecas universitarias

\begin{tabular}{|c|c|c|c|}
\hline Grupo & \multicolumn{3}{|c|}{ Nombre } \\
\hline \multirow{4}{*}{ G1 } & Biblioteca CRAI UPF & Biblioteca UIC Barcelona & Biblioteca UJI \\
\hline & Biblioteca Universitat de Girona & Biblioteca UVic & Biblioteques UAB \\
\hline & CRAI UniBarcelona & CRAI URV & UOC biblioteca \\
\hline & UPC Libraries & & \\
\hline \multirow{7}{*}{ G2 } & Biblioteca CRAI UPO & Biblioteca de la Universidad de Navarra & Biblioteca UAL \\
\hline & Biblioteca UBU & Biblioteca UC & Biblioteca UHU \\
\hline & Biblioteca UJA & Biblioteca Unileon & Biblioteca UNIR \\
\hline & Biblioteca Universidad de Extremadura & Biblioteca UR & Bibliotecas UPSA \\
\hline & Bibliotecas USAL & Biblioteca UA & Biblioteca UCH \\
\hline & Comillas_biblioteca & CRAI Biblioteca UPCT & CSIC - Bib y Arch \\
\hline & Rebiun & UAM_Biblioteca & \\
\hline G3 & Universitat Ramon Llull (URL) & & \\
\hline G4 & Biblioteca de la UCO & & \\
\hline G5 & Biblioteca UPM & Biblioteca Univ Alcalá & BURJC \\
\hline \multirow{2}{*}{ G6 } & Biblio_UM & Biblioteca UMH & $B \cup O$ \\
\hline & UCAM Biblioteca & & \\
\hline G7 & Biblioteca Unizar & & \\
\hline G8 & Biblioteca US & UNIA & \\
\hline G9 & Biblioteca UCLM & & \\
\hline G10 & Biblioteca ULL & Biblioteca ULPGC & Biblioteques UVEG \\
\hline G11 & BiblioUPV & & \\
\hline G12 & Biblioteca Universidad Loyola Andalucía & BibliotecasUSPCEU & \\
\hline G13 & Biblioteca CRAI UEM & & \\
\hline G14 & BiblioComplutense & & \\
\hline G15 & Biblioteca_uc3m & & \\
\hline G16 & BibliotecaUNED & & \\
\hline G17 & BibliotecaUGR & & \\
\hline G18 & Biblioteca_UMA & & \\
\hline G19 & Biblioteca UCA & & \\
\hline G20 & IE Library & UPV/EHU Biblioteka & \\
\hline G21 & Biblioteca UIB & & \\
\hline
\end{tabular}

emiten a través de Twitter) como la forma (imagen, texto o vídeo) de los tweets originales publicados por las cuentas de las bibliotecas universitarias analizadas, buscando diferencias significativas entre estas dos variables. Para ello, del universo total de tweets originales (24.508), se seleccionó una muestra representativa de 379 tweets para el análisis. A continuación se presentan las frecuencias resultantes del análisis de esta muestra (tablas $3,4,5$ y 6 ).
Tabla 3. Frecuencias de tweets en función del formato

\begin{tabular}{|l|c|c|}
\hline Formato del tweet & Frecuencia & Porcentaje \\
\hline Imagen & 237 & 62,5 \\
\hline Texto & 137 & 36,1 \\
\hline Vídeo & 5 & 1,3 \\
\hline Total & 379 & 100 \\
\hline
\end{tabular}

\section{Análisis descriptivo de los tweets originales publicados}

De los tweets analizados, la mayoría de ellos contenía una imagen (62,5\%). El vídeo como recurso de apoyo al tweet aparece en el 1,3\% de los mensajes y tan sólo texto, sin recurso visual de apoyo, el $36,1 \%$.

En cuanto al contenido, tan sólo uno, el de la información de servi-
Tabla 4. Frecuencias de tweets en función de su contenido

\begin{tabular}{|l|c|c|}
\hline \multicolumn{1}{|c|}{ Tipo de contenido } & Frecuencia & Porcentaje \\
\hline Información de servicio & 127 & 33,5 \\
\hline Difusión de investigación/artículos & 66 & 17,4 \\
\hline Difusión de actualidad ajena a la universidad & 61 & 16,1 \\
\hline Adquisición de fondos bibliográficos & 55 & 14,5 \\
\hline Altavoz de actividades culturales y académicas de la universidad & 35 & 9,2 \\
\hline Otros & 35 & 9,2 \\
\hline Total & 379 & 100 \\
\hline
\end{tabular}


cio, destaca sobre el resto, con un tercio del total de mensajes emitidos. Para otras tres categorías los porcentajes son muy similares: difusión de investigación y artículos, 17,4\%; difusión de actualidad ajena a la universidad, 16,1\%; y adquisición de fondos bibliográficos, $14,5 \%$. Tan sólo el contenido relacionado con la actividad de la propia universidad está a cierta distancia en las preferencias (9,2\%), el mismo porcentaje de la categoría "otros".

\section{Tipo de contenido por meses}

Tabla 5. Tipo de contenido por meses (porcentajes)

\begin{tabular}{|c|c|c|c|c|c|c|}
\hline $\begin{array}{l}\text { Etiquetas } \\
\text { de fila }\end{array}$ & $\begin{array}{l}\text { Adquisición y } \\
\text { difusión de fondos } \\
\text { bibliográficos }\end{array}$ & $\begin{array}{l}\text { Altavoz de actividades } \\
\text { culturales y académi- } \\
\text { cas de la universidad }\end{array}$ & $\begin{array}{l}\text { Difusión de } \\
\text { actualidad no } \\
\text { universitaria }\end{array}$ & $\begin{array}{l}\text { Difusión de } \\
\text { investigación, } \\
\text { artículos y tesis }\end{array}$ & $\begin{array}{l}\text { Información de servicio } \\
\text { sobre la biblioteca y } \\
\text { recursos bibliográficos }\end{array}$ & Otros \\
\hline \multicolumn{7}{|c|}{2017} \\
\hline Sep & 9,38 & 18,75 & 15,63 & 18,75 & 34,38 & 3,13 \\
\hline Oct & 15,38 & 11,54 & 19,23 & 11,54 & 38,46 & 3,85 \\
\hline Nov & 9,68 & 12,90 & 32,26 & 9,68 & 35,48 & 0,00 \\
\hline Dic & 13,79 & 10,34 & 10,34 & 24,14 & 24,14 & 17,24 \\
\hline \multicolumn{7}{|c|}{2018} \\
\hline Ene & 13,51 & 10,81 & 13,51 & 16,22 & 32,43 & 13,51 \\
\hline Feb & 25,00 & 5,56 & 19,44 & 11,11 & 33,33 & 5,56 \\
\hline Mar & 18,92 & 13,51 & 8,11 & 13,51 & 29,73 & 16,22 \\
\hline Abr & 20,00 & 13,33 & 13,33 & 26,67 & 26,67 & 0,00 \\
\hline May & 20,45 & 6,82 & 15,91 & 6,82 & 43,18 & 6,82 \\
\hline Jun & 2,56 & 0,00 & 17,95 & 38,46 & 28,21 & 12,82 \\
\hline Jul & 10,34 & 0,00 & 17,24 & 13,79 & 44,83 & 13,79 \\
\hline Ago & 11,11 & 11,11 & 0,00 & 22,22 & 22,22 & 33,33 \\
\hline $\begin{array}{l}\text { Total } \\
\text { general }\end{array}$ & 14,51 & 9,23 & 16,09 & 17,41 & 33,51 & 9,23 \\
\hline
\end{tabular}

Se encontraron diferencias significativas en el contenido de los tweets en función de los meses en los que se publicaron $\left[\chi^{2}(55)=74,746, p<0,05\right]$. La información de servicio es la más relevante para las universidades en cuanto al tema de los tweets durante todos los meses de año, destacando mayo $(43,18 \%)$ y julio $(44,83 \%)$, coincidiendo con períodos de exámenes. De hecho, la mayor parte de los mensajes de esta categoría se refiere a horarios de la biblioteca y espacio disponible. Los temas relacionados con los fondos bibliográficos de la propia universidad adquieren mayor relevancia durante febrero (25\%), mayo $(20,45 \%)$ y abril $(20 \%)$. Los meses en los que más se difunde la actividad cultural y académica de la universidad son septiembre (18,75\%), marzo (13,53\%) y abril $(13,33 \%)$. Respecto a la difusión de la investigaciones, artículos y tesis destaca durante los meses de abril $(26,67 \%)$ y, sobre todo, junio $(38,46 \%)$, el único mes en el que la información de servicio público no es la más difundida (con la excepción de agosto, cuando domina la categoría "otros").

\section{Relación entre el contenido del tweet y su formato}

Se analizó también la relación entre el formato del tweet (con vídeo, con imagen o sólo texto) y su contenido, encontrando diferencias significativas $\left[\chi^{2}(10)=19,058 ; p<0,05\right]$. La imagen es el tipo de formato más utilizado independientemente del tipo de contenido del tweet, pero se utiliza comparativamente más especialmente cuando el mensaje a difundir trata sobre la adquisición de fondos bibliográficos $(80 \%$ de imágenes comparado con el $20 \%$ de texto y $0 \%$ de vídeo). El texto es el segundo recurso más utilizado. Los tweets que contienen sólo texto se reparten de manera relativamente homogénea en los diferentes tipos de contenido de las publicaciones, siendo la difusión de artículos de investigación en donde más se utilizan $(48,5 \%)$ y la adquisi-

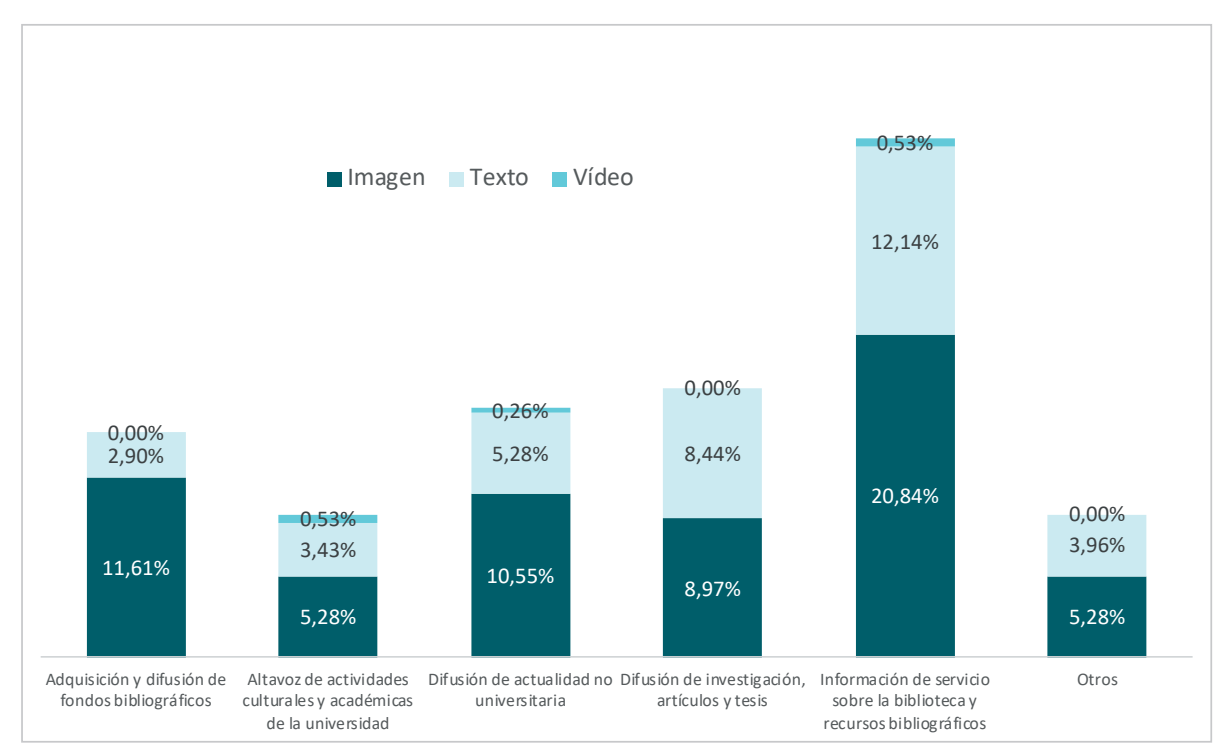

Figura 2. Contenido por formato de tweet (porcentajes sobre el total general). 
ción de fondos bibliográficos en donde menos (20\%). Por último, el vídeo se utiliza muy poco, aunque cuando se recurre a él destaca su uso en la información de actividades culturales y académicas de la universidad $(5,7 \%)$, seguido de información de servicio o la difusión de actualidad ajena a la universidad (1,6\% cada uno).

\section{Engagement en función del formato del tweet}

El engagement de los tipos de tweet Tabla 6. Engagement en función del formato del tweet presentó diferencias significativas en función del formato utilizado $[F(2)=4,29$; $p=0,014]$, siendo mayor en los tweets que contenían imágenes ( $m=0,18 \%$,

\begin{tabular}{|l|c|c|c|c|}
\cline { 2 - 5 } \multicolumn{1}{c|}{} & Imagen & Texto & Vídeo & Total general \\
\hline Promedio de engagement & $0,18 \%$ & $0,09 \%$ & $0,13 \%$ & $\mathbf{0 , 1 5 \%}$ \\
\hline
\end{tabular}
$\mathrm{ds}=0,32 \%)$ seguido de los tweets que contenían vídeo $(\mathrm{m}=0,13 \%, \mathrm{ds}=0,20)$, dejando en último lugar en cuanto al engagement generado a los tweets que sólo mostraban texto $(m=0,09 \%, d s=0,16 \%)$. El engagement medio que obtienen las bibliotecas en cada una de las categorías de su formato de tweet puede considerarse elevado dentro las medias de la red social objeto de estudio (Kell, 2019).

\section{Engagement en función del contenido del tweet}

No se encontraron diferencias significativas en el engagement de los tweets en función de su contenido $[F(5)=0,54$; $p=0,745]$, a pesar de que el mayor engagement lo obtienen los tweets que tratan la adquisición de fondos bibliográficos ( $m=0,19 \%$, ds $=0,25 \%)$ $y$ el menor engagement aparece en los tweets que tratan la difusión de actualidad ajena a la universidad ( $m=0,12 \%$, $\mathrm{ds}=0,18 \%$ ) junto con los tweets que tienen como contenido la difusión de investigaciones, artículos y tesis doctorales $(m=0,12 \%, d s=0,46 \%)$. En este caso también se pueden considerar altos los niveles de enga-

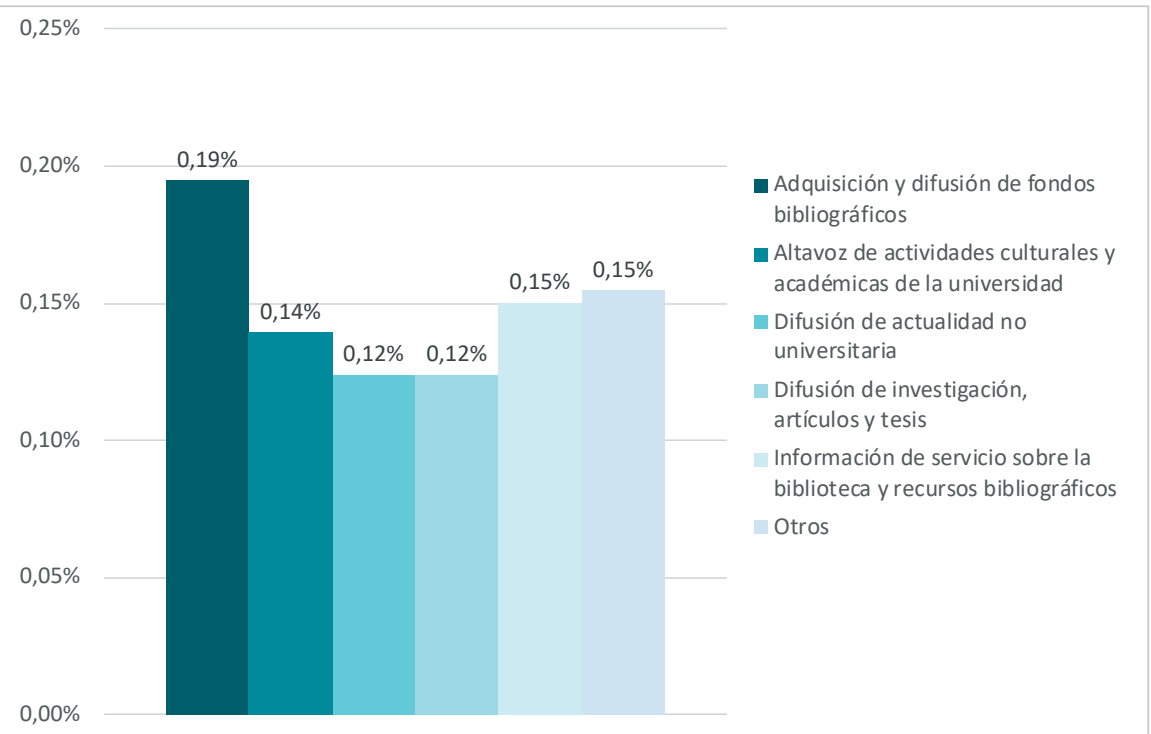

Figura 3. Engagement medio por contenido del tweet gement medio que obtienen los diferentes tipos de tweet (Kell, 2018).

\section{Interacción del formato del tweet sobre el engagement en función del contenido}

Mientras que el formato del tweet presenta efectos significativos en el engagement $[F(2)=0,93 ; p<0,05]$, se comprobó que el tipo de contenido no tenía efectos significativos sobre el mismo $[F(5)=0,19 ; p=0,96]$. De la misma manera, se comprobó que la interacción doble (formato del tweet*tipo de contenido) tampoco tenía efectos significativos en el engagement $[F(7)=0,43 ; p=0,88]$. Es decir, los resultados mostraron que el efecto del formato del tweet sobre el engagement es independiente del contenido del mismo.

\section{Conclusiones y discusión}

Una vez analizada la actividad y el uso que las bibliotecas universitarias españolas hacen de Twitter se puede concluir que, en cuanto a las características de esta red de bibliotecas, el estudio constata que se trata de una red relativamente cohesionada formada por un solo componente. Es decir, que cada uno de los usuarios de la red (ya sean bibliotecas o no) mantiene numerosas interacciones con el resto de usuarios de la misma. Por otro lado, sí se pueden encontrar comunidades que interaccionan en mayor medida por cuestiones de cercanía geográfica, de lengua común o puntuales temas de interés compartido, como puede ser la difusión de la publicación de resultados de investigación interuniversitaria.

Esto se cumple en la mayor parte de los grupos de interacción que se forman dentro de esta red general, aunque hay grupos de bibliotecas cercanas entre sí que no interactúan con bibliotecas de universidades más lejanas. Sólo en el caso de las bibliotecas catalanas (y en menor medida las madrileñas) el criterio geográfico parece influir para estrechar esta relación. En el resto, se forman subgrupos de interacción con universidades muy alejadas geográficamente en los que el nexo de unión son los temas compartidos, por lo que es interesante comprobar que el uso de Twitter permite la posibilidad de compartir conocimiento entre universidades alejadas geográficamente (uno de los objetivos con los que nació internet). En esta relación más o menos estrecha influye también la pertenencia de los centros a un determinado consorcio de bibliotecas, no siempre de la misma Comunidad Autónoma, con lo que Twitter se muestra como un elemento útil a su vez para cohesionar estas redes previamente creadas. 
Dentro de la red, los usuarios más relevantes son aquellos con un mayor índice de centralidad (eigenvector centrality). En este caso los usuarios más influyentes son siempre bibliotecas universitarias, además de la biblioteca del Consejo Superior de Investigaciones Científicas (CSIC). Ningún otro tipo de usuario personal o institucional tiene una posición más predominante dentro de la red. Las bibliotecas más relevantes (las de las universidades Pablo de Olavide de Sevilla, Barcelona, Córdoba, Zaragoza, Castilla-La Mancha, Cantabria, Murcia, Navarra y Loyola Andalucía) pertenecen a diferentes grupos dentro de la red, lo que genera la cohesión de la misma. Al no estar los elementos más influyentes concentrados, la red es más homogénea y la información y el conocimiento se difunden a lo largo de ella de mejor manera.

A la hora de valorar las bibliotecas de las universidades como "puentes de la información y del conocimiento" (betweenness centrality), la Universitat Ramon Llull aparece de forma muy destacada con respecto a todas los demás. Resulta relevante cómo, aunque no es un elemento con alta influencia en la red, sí que lo es a la hora de conectar usuarios. Esto, como se puede observar en los datos recogidos en la tabla 1, lo consigue, en parte, gracias a la gran cantidad de menciones, respuestas y retweets que realiza (i.e., nivel de out-degree). En este caso la biblioteca de esta universidad es la que en mayor medida cumple con la función de conectar elementos. La otra biblioteca que cumple con esta función de forma destacada y no aparece entre las más influyentes es la de la Universitat Oberta de Catalunya.

Respecto a los actores, Rebiun, la institución que agrupa a las bibliotecas, no genera ni difunde información. Es muy mencionada pero no menciona, por lo que no establece una función de mantenimiento y crecimiento de la red. Las bibliotecas, sin embargo, sí cumplen con una función de mantenimiento de la red y de fomento del diálogo a través de los altos niveles de menciones realizadas. Un estudio similar centrado en universidades estadounidenses de tamaño medio (Yep et al., 2017) ya identificó a las bibliotecas como participantes activos en los intercambios universitarios en Twitter. En el caso español estudiado, además de las bibliotecas de las universidades destaca la del CSIC, la institución más importante de España en el campo de la investigación científica.

Por último, en cuanto a los contenidos de los tweets, un tercio de los mensajes enviados por las bibliotecas españolas en el período analizado tiene por objeto dar información de servicio público (fundamentalmente horarios, y especialmente en los meses de exámenes de mayo y julio, cuando suponen casi la mitad del total de mensajes). Este tipo de mensajes, que podrían generar poco debate y por tanto poca difusión por parte de los receptores, puede contribuir a explicar los bajos niveles out-degree. En el resto de tweets se encuentra una presencia muy similar de la difusión de investigación y artículos, la actualidad no universitaria o los fondos bibliográficos y, en menor medida, de altavoz de las actividades de la universidad matriz. Como conclusión, por tanto, se podría decir que la política de comunicación se suele centrar en la información operativa y práctica para los estudiantes, lo cual parece una buena idea teniendo en cuenta que, como encontraron Stvilia y Gibradze (2017), esa es la información más apreciada por los estudiantes.

A su vez, la imagen es el recurso de formato más utilizado independientemente de cuál sea el contenido del tweet, pero especialmente para difundir fondos bibliográficos (portada del libro). Esto es particularmente relevante porque, como muestran los resultados, el tipo de formato es el único factor que predice el engagement de los tweets. En concreto, los tweets que incluyen imágenes (comparados con los tweets que incluyen vídeos y especialmente con los que sólo tienen texto) generan un engagement significativamente mayor independientemente de su contenido. Se puede concluir, por tanto, que los esfuerzos que hacen las bibliotecas en Twitter por añadir una fotografía a sus tweets parece que tiene recompensa, por mucho que esta imagen en ocasiones parezca no aportar información adicional. Este presunto poder de la imagen a la La imagen es el recurso de formato más utilizado por las bibliotecas en Twitter independientemente de cuál sea el contenido del tweet, pero especialmente para difundir fondos bibliográficos

hora de generar engagement en Twitter es, además, consistente con investigaciones previas realizadas por la propia plataforma (Rogers, 2014), en la que los resultados muestran que añadir imágenes es la estrategia (comparada con añadir links, hashtags, vídeos, etc.) que más engagement provoca.

Por otra parte, no existen diferencias significativas entre el contenido del tweet y su engagement. Este resultado contrasta con un estudio previo sobre el uso de Twitter por parte de seis bibliotecas académicas estadounidenses en el que se encontró que los tweets relacionados con los servicios de apoyo al estudio y el mantenimiento de conexiones con la comunidad bibliotecaria eran los que más retweets y favoritos recibían (Stvilia; Gibradze, 2014). Esta discrepancia podría deberse al menos a dos motivos:

- la gran diferencia en magnitud del universo analizado (6 universidades frente a las 59 analizadas en el presente estudio); 
- la medida empleada para evaluar la repercusión de los tweets se calculó de manera ligeramente diferente. En concreto, mientras que el estudio de Stvilia; Gibradze (2014) mide la repercusión teniendo en cuenta únicamente el número simple de retweets y favoritos, el presente estudio utiliza, como se ha explicado previamente, una medida más válida como es la del engagement (Herrera-Torres et al., 2017).

\section{Referencias}

Aharony, Noa (2010). "Twitter use in libraries: An exploratory analysis". Journal of web librarianship, v. 4, n. 4, pp. $333-350$. https://doi.org/10.1080/19322909.2010.487766

Al azar (2019). Números aleatorios y números al azar.

https://bit.ly/22HLcZR

Al-Daihani, Sultan M.; Abrahams, Alan (2016). "A text mining analysis of academic libraries' tweets". The journal of academic librarianship, v. 42, n. 2, pp. 135-143.

https://doi.org/10.1016/j.acalib.2015.12.014

Arroyo-Vázquez, Natalia (2018). "Interacción en las páginas en Facebook de las bibliotecas universitarias españolas”. EI profesional de La información, v. 27, n. 1, pp. 65-74.

https://doi.org/10.3145/epi.2018.ene.06

Ayiah, Efua-Mansa; Kumah, Cynthia-Henewaa (2011). "Social networking: a tool to use for effective service delivery to clients by African libraries". In: World library and information congress: $77^{\text {th }}$ IFLA general conference and assembly. https://www.ifla.org/past-wlic/2011/183-ayiah-en.pdf

Bodnar, Jonathan; Doshi, Ameet (2011). "Asking the right questions: A critique of Facebook, social media, and libraries". Public services quarterly, v. 7, n. 3-4, pp. 102-110.

https://doi.org/10.1080/15228959.2011.623594

Booker, Latrice; Bandyopadhyay, Subir (2013). "How academic libraries can leverage social networking to popularize their services: An empirical study". Journal of the Indiana Academy of the Social Sciences, v. 16, v. 2.

https://bit.ly/2VRWMoe

Bouttier, Jérémie; Di-Francesco, Philippe; Guitter, Emmanuel (2003). "Geodesic distance in planar graphs". Nuclear physics $B$, v. 663, n. 3, pp. 535-567.

https://doi.org/10.1016/S0550-3213(03)00355-9

Casey, Michael E.; Savastinuk, Laura C. (2007). Library 2.0: A guide to participatory library service. Information Today, Inc. ISBN: 9781573872973

Castillo-Díaz, Ana; Herrera-Morillas, José-Luis (2014). Nuevas fórmulas de comunicación con los usuarios de las bibliotecas universitarias. Historia y comunicación social, v. 19, pp. 813-820.

https://doi.org/10.5209/rev_HICS.2014.v19.45004

Charnigo, Laurie; Barnett-Ellis, Paula (2007). "Checking out Facebook.com: The impact of a digital trend on academic libraries". Information technology and libraries, v. 26, n. 1, pp. 23-34.

https://doi.org/10.6017/ital.v26i1.3286

Clauset, Aaron; Newman, Mark. E. J.; Moore, Cristopher (2004). "Finding community structure in very large networks". Physical Review E, v. 70, n. 6, p. 66111 (6 pp.). https://doi.org/10.1103/PhysRevE.70.066111

Connell, Ruth-Sara (2009). "Academic libraries, Facebook and MySpace, and student outreach: A survey of student opinion". Portal: Libraries and the academy, v. 9, n. 1, pp. 25-36.

https://doi.org/10.1353/pla.0.0036

Connor, Elisabeth (2007). "Medical librarian 2.0". Medical reference services quarterly, v. 26, 1, pp. 1-15. https://doi.org/10.1300/J115v26n01_01

CRUE (2017). Bibliotecas. Red de Bibliotecas Universitarias Españolas. CRUE, Universidades Españolas https://bit.ly/2BKT17K

Curran, Kevin; Murray, Michelle; Norrby, David-Stephen; Christian, Martin (2006). "Involving the user through library 2.0". New review of information networking, v. 12, n. 1-2, pp. 47-59.

https://doi.org/10.1080/13614570601136263

Del-Bosque, Darcy; Leif, Sam A.; Skarl, Susie (2012). "Libraries atwitter: Trends in academic library tweeting". Reference services review, v. 40, n. 2, pp. 199-213.

https://doi.org/10.1108/00907321211228246 
Dickson, Andrea; Holley, Robert P. (2010). "Social networking in academic libraries: The possibilities and the concerns". New library world, v. 111, n. 11/12, pp. 468-479.

http://doi.org/10.1108/03074801011094840

Emery, Katie; Schifeling, Todd (2015). "Libraries using Twitter better: Insights on engagement from food trucks". In: $A C R L$ 2015 Proceedings, pp. 450-458.

https://bit.ly/2KgdbvU

Facebook (2019). "Información de la empresa”. Facebook. Newsroom. http://bit.do/eSPTQ

Glazer, Harry (2012). “'Likes' are lovely, but do they lead to more logins?: Developing metrics for academic libraries' Facebook pages". College and research libraries news, v. 73, n. 1, pp. 18-21.

https://doi.org/10.5860/crln.73.1.8688

González-Fernández-Villavicencio, Nieves (2007). “Biblioteca 2.0 en España (el camino recorrido)”. Boletín de la Asociación Andaluza de Bibliotecarios, n. 22, n. 86-87, pp. 29-46.

https://bit.ly/2V5jTGN

Grande-González, Pilar; De-la-Fuente-Redondo, Pablo (2012). "Bibliotecas universitarias españolas en la web social”. EI profesional de la información, v. 21, n. 6, pp. 577-584.

https://doi.org/10.3145/epi.2012.nov.04

Harrison, Amanda; Burress, Rene; Velásquez, Sarah; Schreiner, Lynnette (2017). "Social media use in academic libraries: A phenomenological study". The journal of academic librarianship, v. 43, n. 3, pp. 248-256.

https://doi.org/10.1016/j.acalib.2017.02.014

Herrera-Morillas, José-Luis; Castillo-Díaz, Ana (2011). “Bibliotecas universitarias 2.0. El caso de España”. Investigación bibliotecológica, v. 25, n. 55, pp. 175-200.

https://bit.ly/2BTWBNd

Herrera-Torres, Juan-Carlos; Pérez-Tur, Fernando; García-Fernández, Jerónimo; Fernández-Gavira, Jesús (2017). “El uso de las redes sociales y el engagement de los clubes de la Liga Endesa ACB". Cuadernos de psicología del deporte, v. 17, n. 3, pp. 175-182.

https://revistas.um.es/cpd/article/view/313981

Holmberg, Kim; Huvila, Isto; Kronqvist-Berg, Maria; Widén-Wulff, Gunilla (2009). "What is library 2.0?". Journal of documentation, v. 65, n. 4, pp. 668-681.

https://doi.org/10.1108/00220410910970294

Howard, Heather A.; Huber, Sarah; Carter, Lisa V.; Moore, Elizabeth-Anne (2018). "Academic libraries on social media: Finding the students and the information they want". Information technology and libraries, v. 37, n. 1, pp. 8-18. https://doi.org/10.6017/ital.v37i1.10160

Jain, Priti (2014). "Application of social media in marketing library; information services: A global perspective". International journal of academic research and reflection, v. 2, n. 2, pp. 62-75.

https://bit.ly/2YkpajT

Kell, Laura-Andrea (2019). "How to increase rates of engagement on Twitter". ITP live, 23 May. https://bit.ly/2CE6VtH

Laudano, Claudia-Nora; Corda, María-Cecilia; Planas, Javier A.; Kessler, María-Inés (2016). "Los usos de la red social Facebook por parte de bibliotecas universitarias argentinas. Reflexiones en torno a las dinámicas comunicativas en la web 2.0". Revista interamericana de bibliotecología, v. 39, n. 1, pp. 23-37.

https://doi.org/10.17533/udea.rib.v39n1a05

Lozares, Carlos (1996). "La teoría de redes sociales". Papers. Revista de sociologia, v. 48, pp. 103-126. https://doi.org/10.5565/rev/papers/v48n0.1814

Maness, Jack M. (2006). “Library 2.0 theory: Web 2.0 and its implications for libraries”. Webology, v. 3, n. 2. https://bit.ly/2SRXG7d

Marcos, Mari-Carmen (2009). La biblioteca en la web 2.0. DuocUC.

http://eprints.rclis.org/14849

Margaix-Arnal, Dídac (2007). “Conceptos de web 2.0 y biblioteca 2.0: origen, definiciones y retos para las bibliotecas actuales". El profesional de la información, v. 16, n. 2, pp. 95-106.

https://doi.org/10.3145/epi.2007.mar.01 
Margaix-Arnal, Dídac (2008). “Las bibliotecas universitarias y Facebook: cómo y por qué estar presentes”. El profesional de la información, v. 17, v. 6, pp. 589-602.

https://doi.org/10.3145/epi.2008.nov.02

Marquina, Julián (2018). “Cómo conectan las bibliotecas a través de las redes sociales”. JuliánMarquina, 18 enero. https://bit.ly/2BiJUZ6

Martín-Marichal, María-del-Carmen (2017). Las universidades y las bibliotecas universitarias españolas en las redes sociales: 2017.

https://cutt.ly/V38xRA

Miller, Paul (2005). "Web 2.0: building the new library". Ariadne, n. 45.

https://bit.ly/219WsbW

Morales-Vallejo, Pedro (2012). “Tamaño necesario de la muestra: ¿Cuántos sujetos necesitamos?”. Estadística aplicada a las ciencias sociales. Madrid.

https://tinyurl.com/y3qrj9hs

Newman, Mark E. J. (2006). "Modularity and community structure in networks". Proceedings of the National Academy of Sciences of the United States of America, v. 103, n. 23, pp. 8577-8582.

https://doi.org/10.1073/pnas.0601602103

O’Reilly, Tim (2005). “Web 2.0: Compact definition?”. O’Reilly Radar blog, 1 October.

https://oreil.ly/1mFWDLJ

Pérez-Arranz, Fernando (2008). "Los consorcios de bibliotecas universitarias españolas en el ámbito de la edición electrónica". Boletín de la Asociación Andaluza de Bibliotecarios, v. 23, n. 90-91, pp. 11-28.

https://dialnet.unirioja.es/servlet/articulo?codigo $=2866312$

Petit, Joan (2011). "Twitter and Facebook for user collection requests". Collection management, v. 36, n. 4, pp. $253-258$. http://doi.org/10.1080/01462679.2011.605830

Phillips, Abigail L. (2015). "Facebooking it: Promoting library services to young adults through social media”. Public library quarterly, v. 34, n. 2, pp. 178-197.

https://doi.org/10.1080/01616846.2015.1036710

Piñuel-Raigada, José-Luis (2002). “Epistemología, metodología y técnicas del análisis de contenido”. Estudios de sociolingüística, v. 3, n. 1, pp. 1-42.

https://www.ucm.es/data/cont/docs/268-2013-07-29-Pinuel_Raigada_AnalisisContenido_2002_EstudiosSociolinguisticaUVigo. $p d f$

Rodríguez, Rubén-José (2004). Ayuda SPSS Chi cuadrado. Notas metodológicas.

https://bit.ly/2BTWLnN

Rogers, Simon (2014). “What fuels a Tweet's engagement?". Twitter Blog, 10 March.

https://bit.ly/2wM71tN

Sachs, Dianna E.; Eckel, Eduard J.; Langan, Kathleen A. (2011). "Striking a balance: Effective use of Facebook in an academic library". Internet reference services quarterly, v. 16, n. 1-2, pp. 35-54.

https://doi.org/10.1080/10875301.2011.572457

Sheikh, Arslan; Kazim-Ali, Syed; Naseer, Mirza M. (2016). "Use of social media tools by reputed university libraries of the world: A comparative study". Pakistan library and information science journal, v. 47, n. 2, pp. 45-55.

https://www.researchgate.net/publication/311419742_Use_of_social_media_tools_by_reputed_University_libraries_ of_the_world_A_comparative_study

Shulman, Jason; Yep, Jewelry; Tomé, Daniel (2015). "Leveraging the power of a Twitter network for library promotion". Journal of academic librarianship, v. 41, n. 2, pp. 178-185.

https://doi.org/10.1016/j.acalib.2014.12.004

Smith, Mark; Ceni, Arber; Milic-Frayling, Natasa; Shneiderman, Ben; Mendes-Rodrigues, Eduarda; Leskovec, Jure; Dunne, Cody (2010). NodeXL: a free and open network overview, discovery and exploration add-in for Excel 2007/2010/2013/2016, from the Social Media Research Foundation.

https://www.smrfoundation.org

Spiegel, Murray R.; Stephens, Larry J. (2009). Theory and problems of statistics. New York: McGraw-Hill. ISBN: 007 0602816

http://www.fulviofrisone.com/attachments/article/447/Schaum's\%200utline\%20of\%20Statistic.pdf 
Stephens, Michael (cont.) (2007). "Web 2.0, library 2.0, and the hyperlinked library". Serials review, v. 33, n. 4, pp. 253256.

https://doi.org/10.1016/j.serrev.2007.08.002

Stvilia, Besiki; Gibradze, Leila (2014). "What do academic libraries tweet about, and what makes a library tweet useful?". Library \& information science research, v. 36, n. 3-4, pp. 136-141.

https://doi.org/10.1016/j.lisr.2014.07.001

Stvilia, Besiki; Gibradze, Leila (2017). “Examining undergraduate students' priorities for academic library services and social media communication". Journal of academic librarianship, v. 43, n. 3, pp. 257-262.

https://doi.org/10.1016/j.acalib.2017.02.013

Twitter (2019). Nuestra empresa.

https://bit.ly/2Ilts9s

Vassilakaki, Evgenia; Garoufallou, Emmanouel (2015). "The impact of Twitter on libraries: A critical review of the literature". Electronic library, v. 33, n. 4, pp. 795-809.

https://doi.org/10.1108/EL-03-2014-0051

Vaughan, Liwen; Gao, Yijun (2016). "The use of microblogs and social networking services: A comparison between academic libraries of the United States and China". Journal of web librarianship, v. 10, n. 1, pp. 1-13.

https://doi.org/10.1080/19322909.2015.1118366

Wallis, Richard (2007). "Web 2.0 to library 2.0-from debate to reality. A presentation to the CPD25 Conference, 23 April 2007, London. New review of information networking, v. 13, n. 1, pp. 53-64.

https://doi.org/10.1080/13614570701571484

Wan, Gang (Gary) (2011). "How academic libraries reach users on Facebook". College and undergraduate libraries, v. 18, n. 4, pp. 307-318.

https://doi.org/10.1080/10691316.2011.624944

Yang, Jiang; Counts, Scott (2010). "Predicting the speed, scale, and range of information diffusion in Twitter". In: Procs of the $4^{\text {th }}$ Intl conf on weblogs and social media.

https://www.microsoft.com/en-us/research/publication/predicting-speed-scale-range-information-diffusion-twitter

Yep, Jewelry; Brown, Madison; Fagliarone, Gina; Shulman, Jason (2017). “Influential players in Twitter networks of libraries at primarily undergraduate institutions". Journal of academic librarianship, v. 43, n. 3, pp. 193-200.

https://doi.org/10.1016/j.acalib.2017.03.005

Yep, Jewelry; Shulman, Jason (2014). "Analyzing the library's Twitter network: Using NodeXL to visualize impact". College \& research libraries news, v. 75, n. 4, pp. 177-186.

https://doi.org/10.5860/crln.75.4.9101

Young, Scott-Woodward-Hazard; Rossmann, Doralyn (2015). "Building library community through social media”. Information technology and libraries, v. 34, n. 1, pp. 20-37.

https://doi.org/10.6017/ital.v34i1.5625

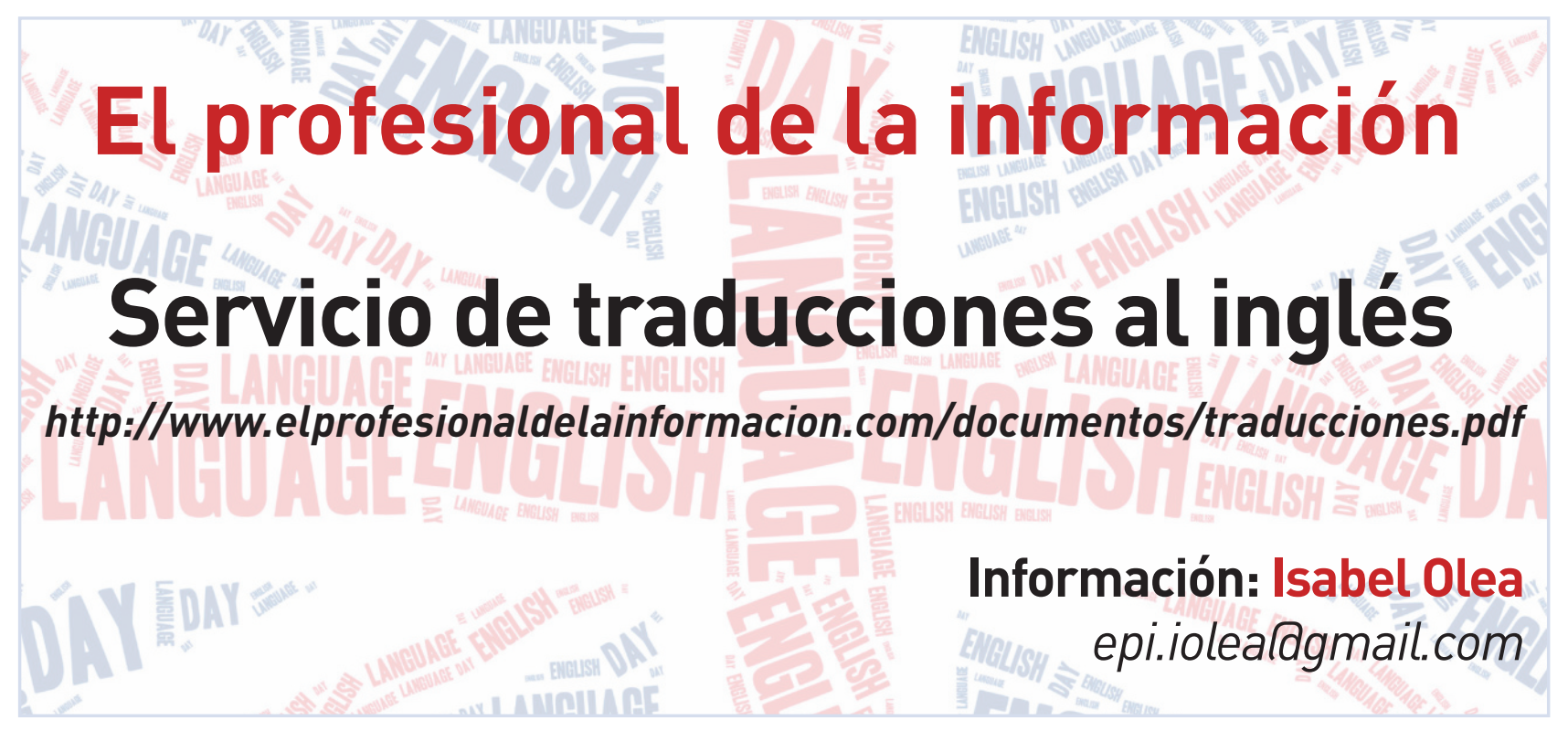

